\title{
Distinct Roles of TLR4 and CD14 in LPS-Induced Inflammatory Responses of Neonates
}

\author{
EVA LEVY, GEORGINA XANTHOU, EFTICHIA PETRAKOU, VASSILIKI ZACHARIOUDAKI, CHRISTOS TSATSANIS, \\ SPYROS FOTOPOULOS, AND MARIETTA XANTHOU
}

\begin{abstract}
Neonatal Immunology Laboratory [E.L., E.P., S.F., M.X.], B'Neonatal Intensive Care Unit (NICU), “Aghia Sophia” Children's Hospital, Athens 115 27, Greece; Cellular Immunology Laboratory [G.X.], Biomedical Research Foundation of the Academy of Athens, Athens 115 27, Greece; Department of Clinical Chemistry [V.Z., C.T.], University of Crete, Crete 71003, Greece
\end{abstract}

\begin{abstract}
During infections, pathogens bind to toll-like receptor (TLR)4 and CD14 receptors and induce cytokine release, leading to inflammation. Here, we investigated TLR4 and CD14 expression on peripheral blood leukocytes (PBLs) and their roles in lipopolysaccharide (LPS)-induced cytokine and chemokine release. Full-term and preterm neonates and adults were studied. PBLs were pretreated with anti-TLR4 - and anti-CD14-blocking antibodies and stimulated with LPS. Cytokine and chemokine levels were measured in supernatants. TLR4, CD14 expression, and LPS-induced CXCL8 release were higher in neonates, possibly contributing to aberrant inflammation. TLR4 blockade resulted in approximately 3-fold greater suppression of LPS-induced CXCL8 release in preterm neonates $(38 \%)$ than in adults $(14 \%)$. CD14 blockade $(\sim 80 \%)$ in neonates induced approximately 3-fold greater inhibition of CXCL8 release, compared with anti-TLR4 $(\sim 30 \%)$. Anti-TLR4 partly (50-60\%) inhibited IL-10 and TNF- $\alpha$, whereas anti-CD14 completely suppressed their release. Our findings reveal that neonates depend more on TLR4 for CXCL8 release. Furthermore, neonatal LPS-induced CXCL8 release, apart from TLR4/CD14-mediated signaling, is regulated by LPS interactions with other TLRs and/or immune receptors. IL-10 and TNF- $\alpha$ release depends on LPS binding not only to CD14/TLR4 but also to CD14 associated with another TLR. Our findings reveal the contribution of TLR4 and CD14 in neonatal cytokine and chemokine release and could aid in design of antagonists to prevent harmful inflammation. (Pediatr Res 66: 179-184, 2009)
\end{abstract}

$\mathrm{N}$ eonates are highly susceptible to Gram-negative bacteria that induce high morbidity and mortality. The principal pathogenic agent involved in neonatal sepsis induced by Gramnegative bacteria is endotoxin lipopolysaccharide (LPS), an essential component of their surface. Defense against pathogens is offered by immune cells, such as granulocytes, monocytes, and dendritic cells (DCs), which express pattern-recognition receptors (PRRs) that recognize specific structures present on microorganisms, termed pathogen-associated molecular patterns (PAMPs) (1). Binding of PAMPs to PRRs triggers antimicrobial responses to combat the infection $(1,2)$.

LPS is one of the best characterized PAMPs that binds to the CD14/toll-like receptor (TLR)4/MD2 complex of PRRs and activates intracellular signaling (3). CD14 binds to LPS

Received November 4, 2008; accepted March 19, 2009.

Correspondence: Marietta Xanthou, M.D., Neonatal Immunology Laboratory, B' NICU, “Aghia Sophia" Children's Hospital, Goudi, Athens 115 27, Greece; e-mail: xanthoumaria@yahoo.com

Supported by a research grant obtained from "Aghia Sophia” Children's Hospital.

Eva Levy and Georgina Xanthou contributed equally to this work. but lacks an intracellular component and is, thus, incapable of signaling. MD2 is a molecule necessary for LPS recognition by TLR4, which also cannot mediate signaling. TLR4, upon LPS binding, leads to intracellular activation of mitogenactivated protein kinase and nuclear factor $-\kappa \mathrm{B}$ that mediate the transcription of proinflammatory cytokine and chemokine genes (4). TLR4 signaling also activates DCs that subsequently present pathogenic peptides to $\mathrm{T}$ lymphocytes and, thus, stimulate T-cell-mediated immunity (5).

The specific roles of TLR4 and CD14 in LPS-induced inflammatory responses by neonatal leukocytes remain not clearly defined. Studies in neonates regarding TLR4 and CD14 expression on peripheral blood monocytes have generated contradictory data, showing either similar $(6,7)$ or lower (8-10) TLR4 and CD14 baseline levels, compared with adults. Additionally, other reports have demonstrated that stimulation of neonatal monocytes with LPS in vitro induces increased TLR4 and CD14 levels, which, however, remain lower than those of adults $(7,9,10)$. In contrast, a recent study by Yerkovich et al. (11) showed increased TLR4 expression upon LPS stimulation in neonates, emphasizing the controversy in the current literature.

During neonatal infections, an exacerbated inflammatory response often occurs inducing the enhanced release of proinflammatory cytokines and chemokines, which may lead to septic shock and death $(12,13)$. In fact, expression levels of TLR4 control the magnitude of the response to LPS. This was shown in transgenic animals overexpressing TLR4 that are more sensitive to endotoxin (14). We, thus, hypothesized that the increased inflammation observed during severe neonatal infections with Gram-negative bacteria is, at least partly, due to an enhanced expression and/or function of CD14 and TLR4 on innate immune cells. To address this, we investigated the expression patterns of TLR4 and CD14 on peripheral blood leukocytes (PBLs) of preterm and full-term neonates. In addition, we studied the roles of TLR4 and CD14 in cytokine and chemokine release by neonatal PBLs after LPS stimulation, using specific blocking antibodies.

Abbreviations: BW, birth weight; DC, dendritic cell; GA, gestational age; LPS, lipopolysaccharide; PAMPs, pathogen-associated molecular patterns; PBLs, peripheral blood leukocytes; PRR, pattern-recognition receptor; TLR, toll-like receptor 


\section{PATIENTS AND METHODS}

Study population. Thirty preterm neonates [birth weight (BW) 1850 (1500-2204 g), gestational age (GA) 33 (32-34 wk)], 30 full-term neonates [BW $3210(3210-3850 \mathrm{~g})$, GA 38 (37-39 wk)], and 30 adults were included in the study. All neonates and adults were healthy and exhibited no signs of hypoxia or asphyxia or infections. Peripheral blood samples were obtained from neonates between the 5th and the 15th postnatal days. We obtained samples only after the 5th day because during the first postnatal days, adaptations of various neonatal functions to the extrauterine life are taking place. Moreover, we did not obtain any samples beyond the 15th postnatal day because we aimed to confine our study to the early neonatal period. Neonates with evidence of major congenital malformations, inborn errors of metabolism, and those who had received immunotherapy were excluded from enrolment. The study was approved by the Hospital Research Ethical Committee and informed written parental consent was obtained before neonates were entered into the study.

Culture and stimulation of peripheral blood samples. Peripheral blood samples were collected from neonates and adults and cultured in flatbottomed 48 -well cell culture plates in $10 \%$ FBS, $1 \%$ penicillin or streptomycin in RPMI 1640 + GlutaMAX (GIBCO BRL, Carlsbad, CA), at $37^{\circ} \mathrm{C}$, and $5 \% \mathrm{CO}_{2}$. Samples were cultured either with medium or with $1000 \mathrm{ng} / \mathrm{mL}$ of LPS (Sigma Chemical Co., St. Louis, MO) for the study of receptor expression and cytokine release. For the investigation of receptor expression, samples were stimulated for $4 \mathrm{~h}$ with LPS. For analysis of cytokine release, samples were stimulated with LPS either for $4 \mathrm{~h}$ (CXCL8, TNF- $\alpha$ ) or for $24 \mathrm{~h}$ (IL-10). An ultrapure preparation of LPS was used in all experiments, because previous reports have indicated that certain LPS preparations contain contaminants that can induce nonspecific signaling $(15,16)$. For blocking studies, whole blood samples were pretreated with anti-TLR2, anti-TLR4 $(1 \mu \mathrm{g} / 100 \mu \mathrm{L}$, eBiosciences, San Diego, CA), and anti-CD14 (5 $\mu \mathrm{L} / 100 \mu \mathrm{L}$, Beckman Coulter, Miami, FL) blocking antibodies, or isotype (Ig) control for $30 \mathrm{~min}$ and then stimulated with $10 \mathrm{ng} / \mathrm{mL}$ LPS. Dose-response studies were performed with anti-TLR4 - and anti-CD14-blocking antibodies to identify the optimal antibody concentration.

Treatment of the THP-1 human monocytic cell line. The THP-1 human monocytic cell line was grown in RPMI 1640 medium, containing $2 \mathrm{mM}$ L-glutamine, $15 \mathrm{mM}$ HEPES, $100 \mathrm{U} / \mathrm{mL}$ penicillin, $0.1 \mathrm{mg} / \mathrm{mL}$ streptomycin, $10 \%$ FBS (Invitrogen, Carlsbad, CA), at $5 \% \mathrm{CO}_{2}$ and $37^{\circ} \mathrm{C}$, as previously described (17). Anti-TLR4-, anti-CD14-blocking antibodies or Ig control were added $1 \mathrm{~h}$ before stimulation with $10 \mathrm{ng} / \mathrm{mL}$ LPS.

Cytokine analysis. Supernatants from cultures of whole blood samples and THP-1 monocytes were collected, centrifuged at $1000 \times \mathrm{g}$ for $5 \mathrm{~min}$, and stored at $-70^{\circ} \mathrm{C}$, until further analysis. Cytokine and chemokine levels were measured by ELISA (R\&D), according to the manufacturer's instructions. The release of CXCL8 and TNF- $\alpha$ from THP- 1 cells was measured by chemiluminescence on Immulite 1000, $24 \mathrm{~h}$ after stimulation, as previously described (18).

Flow-cytometric analysis. Whole blood samples were incubated for 30 min with fluorescently conjugated antibodies to CD14, TLR2, and TLR4 or their respective Ig controls (anti-IgG1 and anti-IgG2a), according to the manufacturer's instructions (eBiosciences). All antibodies were used at 20 $\mu \mathrm{L} / 100 \mu \mathrm{L}$ whole blood. Red blood cells were lysed using VersaLyse (Beckman Coulter). To perform FACS analysis, we used a Coulter cytometer (Epics XL-MCL). Monocytes were identified according to their side scatter properties and $\mathrm{CD} 14^{\text {high }}$ levels of expression. Mean fluorescence intensity (MFI) was determined by subtracting the fluorescence intensity of the Ig isotype controls from that of the specific antibody.

Statistical analysis. Descriptive statistics of cytokine and chemokine levels are given as medians and $25-75 \%$ percentiles. The distribution of values is presented as box plots. The Kruskal-Wallis nonparametric analysis of variance was used for overall comparisons among the groups. Whenever the overall $p$ value was significant, below the 0.05 level $(p<0.05)$, pairwise comparisons were performed using the Mann-Whitney $U$ test.

\section{RESULTS}

Increased TLR4 and CD14 expression on monocytes from neonates. We initially investigated the expression patterns of TLR4 and CD14 on the surface of monocytes in the peripheral blood of healthy preterm and full-term neonates and adults, before and after ex vivo LPS stimulation. Kinetic and doseresponse studies of LPS stimulation were performed to identify the optimal conditions for TLR4 and CD14 expression (data not shown). TLR4 baseline (before LPS stimulation) levels were significantly greater on monocytes from preterm and fullterm neonates, compared with adults $(p=0.004$ and $p=0.016$, respectively), (Fig. 1A). After stimulation with LPS, TLR4 expression was substantially increased in all groups (compared with nonstimulated cells), and it remained significantly greater in full-term neonates compared with adults $(p=0.009$; Fig. $1 A)$. CD14 baseline levels were significantly greater in full-term neonates compared with those of adults $(p<0.0001$; Fig. $1 B)$. After stimulation with LPS, CD14 expression was increased in all groups, and it remained significantly greater in full-term neonates, compared with adults $(p<0.001$; Fig. $1 B)$.

Overall, enhanced TLR4 and CD14 expression was observed in neonatal peripheral blood monocytes and this may participate in the exacerbated inflammatory response often occurring during neonatal infections.

Elevated CXCL8 levels in neonatal whole blood cells upon LPS stimulation. We next investigated the LPS-induced release of CXCL8, TNF- $\alpha$, and IL-10 by PBLs from neonates and adults. In the absence of LPS, CXCL8, TNF- $\alpha$, and IL-10 levels were undetectable in whole blood culture supernatants (Fig. 2). Stimulation of PBLs with LPS resulted in increased levels of CXCL8, TNF- $\alpha$, and IL-10 in all groups studied (Fig. 2). CXCL8 release by PBLs upon LPS stimulation was significantly greater in both preterm and full-term neonates, compared with adults $(p=0.005$ and $p=0.0001$, respectively; Fig. $2 A$ ). No significant differences were observed in the release of TNF- $\alpha$ and IL-10 after LPS stimulation among the groups studied (Fig. $2 B$ and $C$, respectively).

Distinct roles of TLR4 and CD14 in LPS-induced responses from neonatal peripheral blood cells. We subsequently studied the specific roles of TLR4 and CD14 in LPSinduced release of CXCL8, TNF- $\alpha$, and IL-10 from PBLs of neonates and adults. Treatment with anti-TLR4 resulted in partial inhibition of the LPS-induced CXCL8 release from PBLs of preterm and full-term neonates and adults $(38,21$, and $14 \%$,
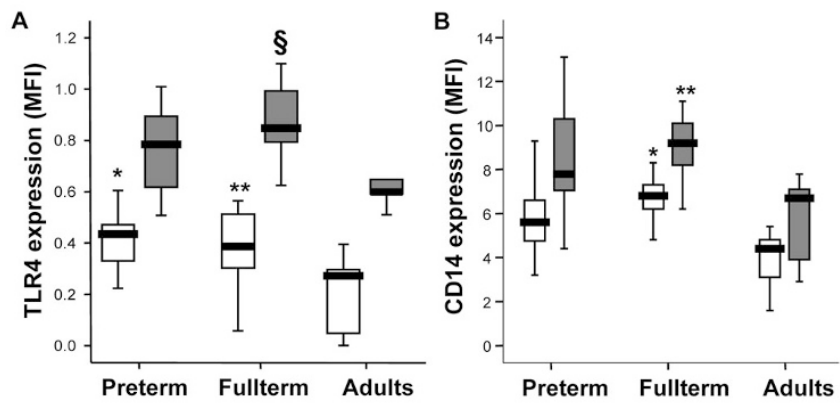

Figure 1. Increased TLR4 and CD14 surface expression in monocytes from neonates. A, TLR4 surface expression was examined on CD14 ${ }^{\text {high }}$ monocytes prior and after stimulation with LPS ex vivo. Baseline TLR4 levels (white bars) were significantly greater in preterm and full-term neonates, compared with adults $(* p=0.004$ and $* * p=0.016$, respectively). After stimulation with LPS (gray bars) TLR4 levels were also significantly greater in full-term neonates, compared with adults $(\$ p=0.009)$. $B$, CD14 surface expression was examined on PBLs at baseline (white bars) and after stimulation with LPS ex vivo (gray bars). Baseline CD14 levels were significantly greater in full-term neonates, compared with adults $(* p<0.0001)$. CD14 levels were also significantly greater in full-term neonates, compared with adults after stimulation with LPS $(* * p<0.0001)$. 

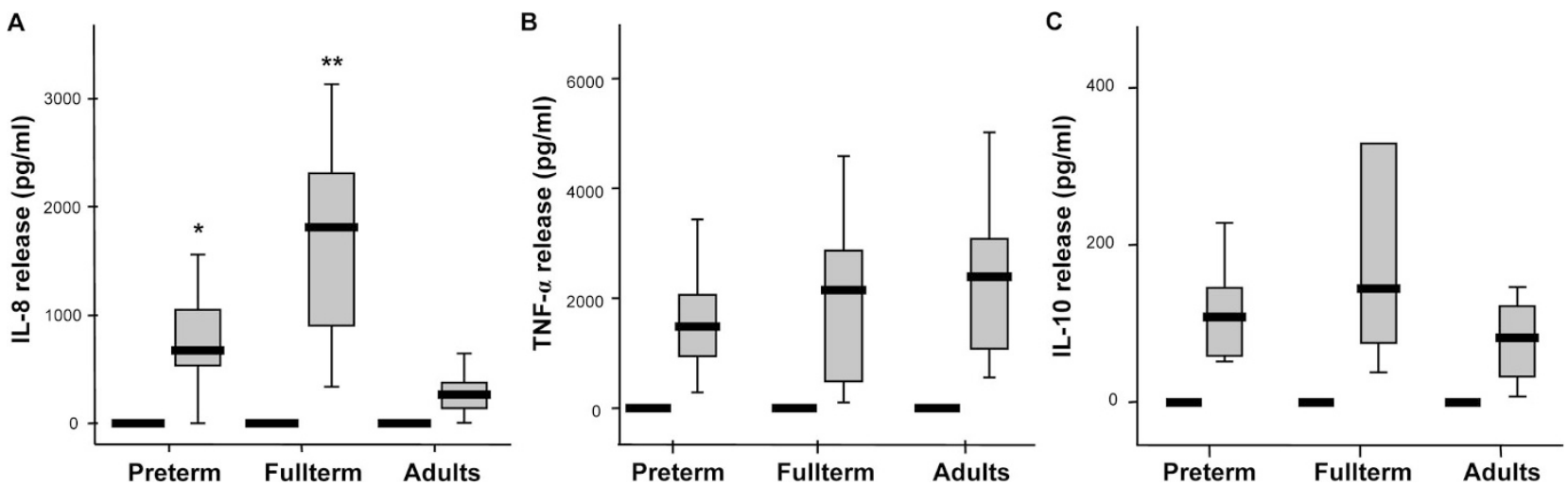

Figure 2. Increased LPS-induced CXCL8 release by neonatal leukocytes. Levels of (A) CXCL8, $(B)$ TNF- $\alpha$, and $(C)$ IL-10 in culture supernatants of PBLs from neonates and adults before (black bars) or after stimulation with LPS ex vivo (gray bars). CXCL8 levels were significantly greater in preterm and full-term neonates, compared with adults $(* p=0.005$ and $* * p=0.0001$, respectively).
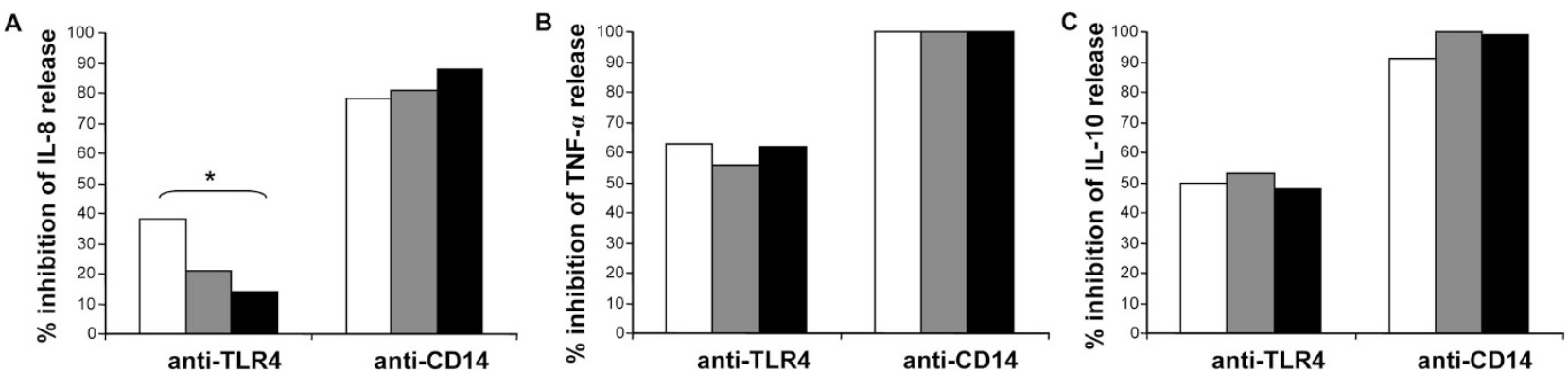

Figure 3. TLR4 and CD14 have distinct roles in LPS-induced responses of neonatal leukocytes. Percent inhibition of LPS-induced (A) CXCL8, $(B)$ TNF- $\alpha$, and $(C)$ IL-10 release after blockade of TLR4 and CD14 in PBLs from preterm (white bars), full-term (gray bars) neonates, and adults (black bars) is shown. The percent inhibition of CXCL8 release upon anti-TLR4 treatment was significantly greater in preterm neonates, compared with adults $(* p=0.001)$.

respectively; Fig. $3 A$ ), compared with treatment with Ig control. Notably, the percent inhibition in CXCL8 release upon antiTLR4 treatment was significantly greater $(\sim 3$-fold $)$ in preterm neonates (38\%), compared with that in adults $(14 \%, p=0.001$; Fig. 3A). Treatment with anti-CD14 also inhibited $(\sim 80 \%)$ the LPS-induced CXCL8 release in all groups studied (Fig. 3A). Interestingly, CD14 blockade $(\sim 80 \%)$ induced approximately a 3 -fold greater inhibition of the CXCL8 release in neonates, compared with TLR4 blockade $(\sim 30 \%)$. Treatment with both anti-TLR4 and anti-CD14 did not result in a further increase in the inhibition of LPS-induced CXCL8 release, compared with treatment with anti-CD14 alone in any of the groups studied.

The LPS-induced TNF- $\alpha$ release was partially inhibited $(\sim 60 \%)$ upon TLR4 blockade, compared with Ig control, in all groups studied (Fig. 3B). In addition, treatment with antiCD14 resulted in complete inhibition $(100 \%)$ of the LPSinduced TNF- $\alpha$ release (Fig. 3B). Similarly to TNF- $\alpha$, the LPS-induced IL-10 release was partially inhibited (preterm neonates $50 \%$, full-term neonates $53 \%$, and adults $48 \%$ ) by TLR4 blockade, whereas anti-CD14 treatment completely $(\sim 100 \%)$ suppressed IL-10 release, compared with Ig control (Fig. 3C). Our data indicate that the LPS-induced TNF- $\alpha$ and IL-10 release depends more on the TLR4/CD14 complex compared with that of CXCL8.

Because LPS-induced CXCL8, TNF- $\alpha$, and IL-10 release was not completely inhibited by anti-TLR4 treatment, we hypothesized that LPS also uses another TLR for signaling. Certain studies have shown that LPS can also bind and signal through TLR2 $(19,20)$. Hence, we next investigated whether TLR2 was involved in LPS-induced responses by neonates. Anti-TLR2 did not inhibit LPS-induced release of CXCL8, TNF- $\alpha$, and IL-10 in any of the groups studied (data not shown), suggesting that TLR2 does not participate in this LPS response.

Our data suggest that IL-10 and TNF- $\alpha$ release depends not only on LPS binding and signaling through the CD14/TLR4 complex but also on CD14 associated with another TLR, whereas CXCL8 release, apart from CD14/TLR4 binding, also depends on LPS interactions with other TLRs and/or immune receptors.

Effects of TLR4 and CD14 blockade on LPS-induced responses in a human monocytic cell line. LPS has been shown to bind to surface receptors present not only on monocytes but also on other leukocytes, such as granulocytes and DCs. Therefore, it is plausible that these cells may have also contributed to the observed effects of TLR4 and CD14 blockade on LPS-induced responses in neonatal PBLs. To determine whether the neonatal PBL responses observed after LPS stimulation were, at least partly, induced by monocytes, we performed a similar set of experiments using the human monocytic THP-1 cell line. THP-1 monocytes have been extensively used for the study of LPS-induced TLR4 signaling $(21,22,23)$. Anti-TLR4 treatment partially inhibited $(17 \%)$ the LPS-induced CXCL8 release, whereas CD14 blockade resulted in a 4-fold greater inhibition (76\%) of CXCL8 release, compared with anti-TLR4 (Fig. 4A). Blockade of both TLR4 

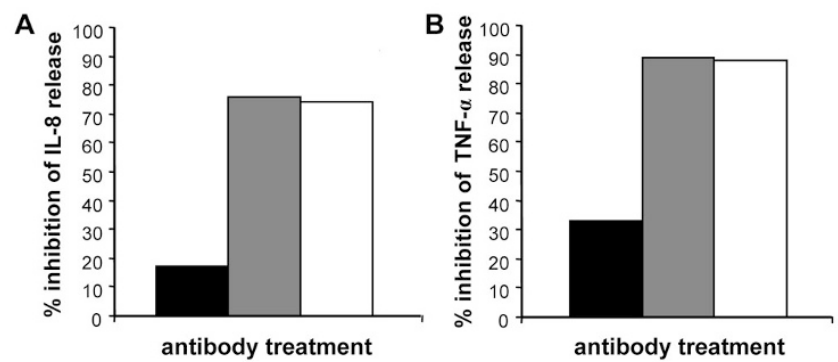

Figure 4. Effects of TLR4 and CD14 blockade on LPS-induced responses by the human THP-1 monocytic cell line. Percent inhibition of LPS-induced $(A)$ CXCL8 and (B) TNF- $\alpha$ release by blockade of either TLR4 (black bars), CD14 (gray bars) or TLR4 and CD14 (white bars) in THP-1 cells is shown.

and CD14 did not result in a further increase in the inhibition of LPS-induced CXCL8 release, compared with treatment with anti-CD14 alone (Fig. $4 A$ ). TNF- $\alpha$ release was partially inhibited by anti-TLR4 and anti-CD14 treatment (33\% and $89 \%$, respectively), compared with Ig control (Fig. 4B). As with CXCL8, blockade of both TLR4 and CD14 did not result in a further increase in the inhibition of LPS-induced TNF- $\alpha$ release, compared with treatment with anti-CD14 alone (Fig. $4 B$ ). These data mirror the ones obtained from neonatal PBLs and suggest that the effects observed on TLR4 and CD14 blockade on LPS-induced responses are mainly mediated by neonatal monocytes.

\section{DISCUSSION}

In this study, we have demonstrated that preterm and full-term neonates express significantly greater levels of TLR4 on peripheral blood monocytes both at baseline and after LPS stimulation, compared with adults. In agreement, Yerkovich et al. (11) demonstrated higher TLR4 levels on peripheral blood monocytes after LPS stimulation in neonates, compared with infants and adults. However, other studies using cord blood samples showed either similar (8) or lower $(6,7)$ TLR4 levels on monocytes after LPS stimulation. We also observed significantly greater CD14 expression at baseline and after LPS stimulation in full-term neonates compared with adults. In previous studies, CD14 baseline levels were found to be either similar $(6,7)$ or lower $(8-10)$ than those of adults. LPS stimulation increased CD14 levels in cord blood monocytes, which, however, remained lower than those of adults $(9,10)$. The aforementioned disparities regarding TLR4 and CD14 expression levels may be due to differences in the LPS concentration used or the time point of analysis, as well as, to the fact that we have used neonatal peripheral blood and not cord blood. Furthermore, because the number of neonates examined in some of the cord blood studies was very small, it may be difficult to reach solid conclusions, taking also into consideration the wide range of normality in neonatal values. Collectively, our data suggest that the increased TLR4 and CD14 expression on LPS stimulation in neonatal monocytes may participate in the exacerbated inflammation, which is often observed during severe infections.

Our findings also demonstrated significantly increased levels of the proinflammatory chemokine CXCL8 upon LPS stimulation of neonatal PBLs, compared with adults. These findings are in agreement with those from several other studies (24-28). In addition, we showed that LPS-induced TNF- $\alpha$ production was similar between neonates and adults, as also demonstrated by other studies $(11,24,29)$. However, other investigators have found lower TNF- $\alpha$ levels by neonatal leukocytes upon LPS stimulation compared with adults $(6,7)$. TNF- $\alpha$ release seems to depend on the dose of LPS used. This was shown in a study by Yan et al., (7) who demonstrated that high LPS concentrations $(10 \mathrm{ng} / \mathrm{mL})$ induced similar TNF- $\alpha$ levels in adults and neonates, whereas, at lower $(0.1 \mathrm{ng} / \mathrm{mL})$ LPS concentrations, TNF- $\alpha$ secretion was significantly lower in newborns. Furthermore, we observed that neonatal PBLs after LPS stimulation produced the anti-inflammatory cytokine IL-10 at levels similar to those of adults. IL-10 levels have been demonstrated to be either similar $(11,24)$ or lower to those of adults (30). Thus, our data indicate that neonates mount a more pronounced proinflammatory response, whereas their anti-inflammatory response is similar to that of adults.

Blockade of TLR4 resulted in only partial inhibition of the LPS-induced CXCL8 release by PBLs from neonates and adults. This suggested that another TLR and/or MD2 may be involved in CXCL8 release after LPS stimulation. Importantly, our data showed that preterm neonates depended more on TLR4 for CXCL8 release (38\% inhibition), compared with full-term neonates and adults (21 and 14\% inhibition, respectively), pointing to distinct signaling pathways. In our effort to identify an alternate LPS receptor, we treated PBLs with anti-TLR2, a receptor considered to mediate LPS responses $(19,20)$. However, blockade of TLR2 did not inhibit LPSinduced CXCL8 release.

Furthermore, our findings revealed that CD14 blockade did not completely inhibit the LPS-induced CXCL8 release from neonatal PBLs, suggesting the presence also of CD14independent mechanisms for LPS responses. In agreement with our data, certain studies have shown that CD14 is not critical for LPS binding (31) and TNF- $\alpha$ production (32) in adult monocytes. However, others support the importance of CD14 in eliciting LPS responses because CD14-deficient mice exhibited impaired LPS uptake (33) and were highly resistant to shock mediated by Gram-negative bacteria (34).

Blockade of both TLR4 and CD14 did not result in a complete suppression of LPS-induced CXCL8 release by neonatal PBLs. This suggests that the LPS-induced CXCL8 release also depends on another receptor. MD2 may be such a receptor and simultaneous blockade of MD2 merits further investigation. Alternatively, apart from the CD14/TLR/MD2 complex, LPS may regulate CXCL8 via binding and signaling through a different immune cell receptor. The receptor of mannose-binding lectin may be such a signaling molecule, as a recent study has demonstrated that mannose-binding lectin enhances LPS-mediated release of proinflammatory cytokines and chemokines by endothelial cells, possibly through binding to its receptor (35).

LPS-induced release of TNF- $\alpha$ and IL-10 by PBLs was only partially inhibited ( $\sim 60$ and $\sim 50 \%$, respectively) by TLR4 blockade in neonates, suggesting that LPS utilizes both TLR4-dependent and TLR4-independent mechanisms. In con- 
trast, CD14 blockade resulted in complete inhibition of both TNF- $\alpha$ and IL-10 release, suggesting that CD14 is essential for these responses. Taken together, our studies revealed that TNF- $\alpha$ and IL-10 release by neonatal PBLs depends on LPS binding to CD14 associated not only with TLR4, but also with another TLR and/or MD2. Studies using blocking of CD14 and TLR4 receptors to determine their effect on cytokine production have been limited in adults $(36,37)$. In a single study in neonates, Karlsson et al. (24) demonstrated that blockade of either CD14 or TLR4 suppressed TNF- $\alpha$ release. However, the investigators used a different approach that involved stimulation of cord blood monocytes with whole Escherichia coli bacteria (24). It is possible that this disparity is due to the different experimental protocols used (i.e. differences in the amounts of E. coli and LPS used). Interestingly, our data indicate that TLR4 plays a more prominent role in LPS-induced release of TNF- $\alpha$ and IL-10, compared with CXCL8. Summarizing our results, we propose a model that describes the distinct roles of TLR4 and CD14 in LPS-induced responses of neonatal PBLs (Fig. 5).

The pathophysiology of an infectious disease may be caused not only by the pathogen involved but also by excessive inflammatory responses, which are sometimes more damaging to the host than the virulence activity of the pathogen that elicited them. Thus, understanding the balance between these two causes of host tissue damage is crucial for the development of appropriate therapeutic strategies. Soluble TLRs or antibodies that block LPS binding to TLRs or to CD14 and MD2 have been used in the treatment of sepsis (38). The findings of our current study have demonstrated increased TLR4 and CD14 expression by neonatal PBLs after LPS stimulation, compared with adults, supporting the importance of TLR4 and CD14 expression levels in regulating the magnitude of the response to LPS. Moreover, our data reveal the
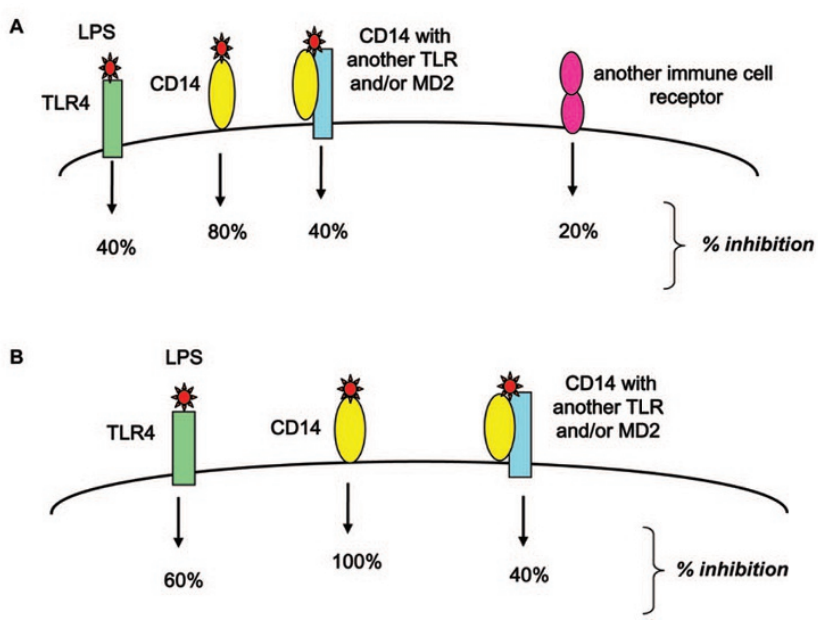

Figure 5. Proposed models for the roles of TLR4 and CD14 in LPS-induced release of (A) CXCL8 and $(B)$ TNF- $\alpha$ and IL-10 by neonatal PBLs. $A$, LPS-induced CXCL8 release depends on TLR4 ( 40\%), CD14 ( 80\%) and CD14 associated with another TLR and/or MD2 ( $\sim 40 \%)$. However, CXCL8 release may also depend on LPS interactions with another immune cell receptor (remaining 20\%). $B$, LPS-induced IL-10 and TNF- $\alpha$ release depends on TLR4 ( $\sim 60 \%)$, on CD14 ( $\sim 100 \%)$, and on CD14 associated with another TLR and/or MD2 (remaining $40 \%$ ). specific roles of TLR4 and CD14 in LPS-induced responses in neonates and suggest that other immune cell receptors may be also involved. Our findings could aid in the design of antagonists against TLR4 and CD14 for blocking or partially blocking the release of specific cytokines or chemokines, which may ultimately prevent harmful and potentially lifethreatening inflammatory responses, induced by Gramnegative bacteria in newborns.

\section{REFERENCES}

1. Medzhitov 2007 Recognition of microorganisms and activation of the immune response. Nature 449:819-826

2. Marodi L 2006 Innate cellular immune responses in newborns. Clin Immunol 118:137-144

3. Dobrovolskaia MA, Vogel SN 2002 Toll receptors, CD14, and macrophage activation and deactivation by LPS. Microbes Infect 4:903-914

4. Lakhani SA, Bogue CW 2003 Toll-like receptor signaling in sepsis. Curr Opin Pediatr 15:278-282

5. Janeway CA 1998 Presidential Address to The American Association of Immunologists. The road less traveled by: the role of innate immunity in the adaptive immune response. J Immunol 161:539-544

6. Levy O, Zarember KA, Roy RM, Cywes C, Godowski PJ, Wessels MR 2004 Selective impairment of TLR-mediated innate immunity in human newborns: neonatal blood plasma reduces monocyte TNF-alpha induction by bacterial lipopeptides, lipopolysaccharide, and imiquimod, but preserves the response to R-848. J Immunol 173:4627-4634

7. Yan SR, Qing G, Byers DM, Stadnyk AW, Al-Hertani W, Bortolussi R 2004 Role of MyD88 in diminished tumor necrosis factor alpha production by newborn mononuclear cells in response to lipopolysaccharide. Infect Immun 72:1223-1229

8. Forster-Waldl E, Sadeghi K, Tamandl D, Gerhold B, Hallwirth U, Rohrmeister K, Hayde M, Prusa AR, Herkner K, Boltz-Nitulescu G, Pollak A, Spittler A 2005 Monocyte toll-like receptor 4 expression and LPS-induced cytokine production increase during gestational aging. Pediatr Res 58:121-124

9. Henneke P, Osmers I, Bauer K, Lamping N, Versmold HT, Schumann RR 2003 Impaired CD14-dependent and independent response of polymorphonuclear leukocytes in preterm infants. J Perinat Med 31:176-183

10. Qing G, Rajaraman K, Bortolussi R 1995 Diminished priming of neonatal polymorphonuclear leukocytes by lipopolysaccharide is associated with reduced CD14 expression. Infect Immun 63:248-252

11. Yerkovich ST, Wikstrom ME, Suriyaarachchi D, Prescott SL, Upham JW, Holt PG 2007 Postnatal development of monocyte cytokine response to bacterial lipopolysaccharide. Pediatr Res 62:547-552

12. Tatad AM, Nesin M, Peoples J, Cheung S, Lin H, Sison C, Perlman J, CunninghamRundles S 2008 Cytokine expression in responses to bacterial antigens in preterm and term infant cord blood monocytes. Neonatology 94:8-15

13. Meng G, Rutz M, Schiemann M, Metzger J, Grabiec A, Schwandner R, Luppa PB, Ebel F, Busch DH, Bauer S, Wagner H, Kirschning SJ 2004 Antagonistic antibody prevents toll-like receptor 2-driven lethal shock-like syndromes. J Clin Invest 113:1473-1481

14. Bihl F, Salez L, Beaubier M, Torres D, Lariviere L, Laroche L, Benedetto A, Martel D, Lapointe JM, Ryffel B, Malo D 2003 Overexpression of toll-like receptor 4 amplifies the host response to lipopolysaccharide and provides a survival advantage in transgenic mice. J Immunol 170:6141-6150

15. Manthey CL, Perera PY, Henricson BE, Hamilton TA, Qureshi N, Vogel SN 1994 Endotoxin-induced early gene expression in $\mathrm{C} 3 \mathrm{H} / \mathrm{HeJ}\left(\mathrm{Lps}^{d}\right)$ macrophages. J Immunol 153:2653-2663

16. Hogan MM, Vogel SN 1988 Production of tumor necrosis factor by rIFN- $\gamma$-primed $\mathrm{C} 3 \mathrm{H} / \mathrm{HeJ}\left(\mathrm{Lps}^{d}\right)$ macrophages requires the presence of lipid A-associated proteins. J Immunol 141:4196-4202

17. Tsatsanis C, Zacharioudaki V, Androulidaki A, Dermitzaki E, Charalampopoulos I, Minas V, Gravanis A, Margioris AN 2005 Adiponectin induces TNF-alpha and IL-6 in macrophages and promotes tolerance to itself and other pro-inflammatory stimuli. Biochem Biophys Res Commun 335:1254-1263

18. Berthier F, Lambert C, Genin C, Bienvenu J 1999 Evaluation of an automated immunoassay method for cytokine measurement using the Immulite Immunoassay system. Clin Chem Lab Med 37:593-599

19. Hirschfeld M, Ma Y, Weis JH, Vogel SN, Weis JJ 2000 Cutting edge: repurification of lipopolysaccharide eliminates signaling through both human and murine toll-like receptor 2. J Immunol 165:618-622

20. Kirschning CJ, Wesche H, Merrill Ayres T, Rothe M 1998 Human toll-like receptor 2 confers responsiveness to bacterial lipopolysaccharide. J Exp Med 188:2091-2097

21. Wu W, Alexis NE, Chen X, Bromberg PA, Peden DB 2008 Involvement of mitogen-activated protein kinases and NFkappaB in LPS-induced CD40 expression on human monocytic cells. Toxicol Appl Pharmacol 228:135-143

22. Li CH, Wang JH, Redmond HP 2006 Bacterial lipoprotein-induced self-tolerance and cross-tolerance to LPS are associated with reduced IRAK-1 expression and MyD88-IRAK complex formation. J Leukoc Biol 79:867-875

23. Zhang FX, Kirschning CJ, Mancinelli R, Xu XP, Jin Y, Faure E, Mantovani A, Rothe M, Muzio M, Arditi M 1999 Bacterial lipopolysaccharide activates nuclear factor-kappaB through interleukin-1 signaling mediators in cultured human dermal endothelial cells and mononuclear phagocytes. J Biol Chem 274:7611-7614 
24. Karlsson H, Hessle C, Rubin A 2002 Innate immune responses of human neonatal cells to bacteria from the normal gastrointestinal flora. Infect Immun 70:6688-6696

25. Berner R, Welter P, Brandis M 2002 Cytokine expression of cord and adult blood mononuclear cells in response to Streptococcuw agalactiae. Pediatr Res 51:304-309

26. Schultz C, Rott C, Temming P, Schlenke P, Moller JC, Bucsky P 2002 Enhanced interleukin-6 and interleukin- 8 synthesis in term and preterm infants. Pediatr Res $51: 317-322$

27. Dembinski J, Behrendt D, Reinsberg J, Bartmann P 2002 Endotoxin-stimulated production of IL-6 and IL-8 is increased in short-term cultures of whole blood from healthy term neonates. Cytokine 18:116-119

28. Powell C, Orsi N, Simpson N, Levene M 2004 Characterisation of the cytokine inflammatory response in LPS stimulated full-term cord blood. J Perinat Med 32:440-445

29. De Wit D, Tonon S, Olislagers V, Goriely S, Boutriaux M, Goldman M, Willems F 2003 Impaired responses to toll-like receptor 4 and toll-like receptor 3 ligands in human cord blood. J Autoimmun 21:277-281

30. Chheda S, Palkowetz KH, Garofalo R, Rassin DK, Goldman AS 1996 Decreased interleukin-10 production by neonatal monocytes and $\mathrm{T}$ cells: relationship to decreased production and expression of tumor necrosis factor-alpha and its receptors. Pediatr Res 40:475-483

31. Corrales I, Weersink AJ, Verhoef J, van Kessel KP 1993 Serum-independent binding of lipopolysaccharide to human monocytes is trypsin sensitive and does not involve CD14. Immunology 80:84-89
32. Lynn WA, Liu Y, Golenbock DT 1993 Neither CD14 nor serum is absolutely necessary for activation of mononuclear phagocytes by bacterial lipopolysaccharide. Infect Immun 61:4452-4461

33. Dunzendorfer S, Lee HK, Soldau K, Tobias PS 2004 TLR4 is the signaling but not the lipopolysaccharide uptake receptor. J Immunol 173:1166-1170

34. Haziot A, Ferrero E, Kontgen F, Hijiya N, Yamamoto S, Silver J, Stewart CL, Goyert SM 1996 Resistance to endotoxin shock and reduced dissemination of gram-negative bacteria in CD14-deficient mice. Immunity 4:407-414

35. Kang HJ, Lee SM, Lee HH, Kim JY, Lee BC, Yum JS, Moon HM, Lee BL 2007 Mannose-binding lectin without the aid of ist associated serine proteases alter lipopolysaccharide-mediated cytokine/chemokine secretion from human endothelial cells. Immunology 122:335-342

36. Andreakos E, Sacre SM, Smith C, Lundberg A, Kiriakidis S, Stonehouse T 2004 Distinct pathways of LPS-induced NF-kB activation and cytokine production in human myeloid and non-myeloid cells defined by selective utilization of MyD88 and Mal/TIRAP. Blood 103:2229-2237

37. Elson G, Dunn-Siegrist I, Daubeuf B, Pugin J 2007 Contribution of toll-like receptors to the innate immune response to Gram-negative and Gram-positive bacteria. Blood 109:1574-1583

38. O'Neill LA 2003 Therapeutic targeting of Toll-like receptors for inflammatory and infectious diseases. Curr Opin Pharmacol 3:396-403 